\title{
Some Properties and Applications of Topp Leone Kumaraswamy Lomax Distribution
}

\author{
Ibrahim Sule ${ }^{1 *}$, Sani Ibrahim Doguwa ${ }^{2}$, Audu Isah $^{3}, \&$ Haruna, M. Jibril ${ }^{4}$ \\ ${ }^{1 \& 2}$ Department of Statistics, Faculty of Physical Sciences, Ahmadu Bello University, Zaria \\ ${ }^{3}$ Department of Statistics, School of Physical Sciences, Federal University of Technology, Minna \\ ${ }^{4}$ Department of Mathematics, Faculty of Physical Sciences, Ahmadu Bello University, Zaria \\ *Corresponding author: ibrahimsule76@yahoo.com
}

Many Statisticians have developed and proposed new distributions by extending the existing distributions. The distributions are extended by adding one or more parameters to the baseline distributions to make it more flexible in fitting different kinds of data. In this study, a new fourparameter lifetime distribution called the Topp Leone Kumaraswamy Lomax distribution was introduced by using a family of distributions which has been proposed in the literature. Some mathematical properties of the distribution such as the moments, moment generating function, quantile function, survival, hazard, reversed hazard and odds functions were presented. The estimation of the parameters by maximum likelihood method was discussed. Three real life data sets representing the failure times of the air conditioning system of an air plane, the remission times (in months) of a random sample of one hundred and twenty-eight (128) bladder cancer patients and Alumina (A12O3) data were used to show the fit and flexibility of the new distribution over some lifetime distributions in literature. The results showed that the new distribution fits better in the three datasets considered.

Keywords: Bladder cancer patients, exponentiated Lomax, maximum likelihood estimation, reversed hazard rate function, Topp Leone Kumaraswamy-G

\section{Introduction}

Researchers have been working on developing new probability distributions by adding parameter or parameters to the standard distributions to generalize them and make them more flexible in modeling real life data sets. The addition of one or more parameters to the standard models has made the generalized models gain wide applications in areas such as Insurance, Finance, Medicine, Environmental Sciences, etc. The Lomax (Pareto type II) distribution is a continuous probability distribution with a heavy tail having one shape and one scale parameters. The distribution was introduced by Lomax (1954) to analyse business failure data. Researchers have proposed different extensions of the Lomax distribution such as the extended Lomax by Lemonte and Cordeiro, (2013), gamma-Lomax by Cordeiro et al., (2013), exponentiated Lomax by El-Bassiouny et al., (2015), The Transmuted Weibull Lomax Distribution by Ahmed et al., (2015), Weibull Lomax by Tahir et al., (2015), 
Gompertz Lomax by Oguntunde et al., (2017).

The two parameter Lomax distribution has the cumulative distribution function (cdf) and probability density function (pdf) given respectively as:

$H(x ; \beta, \sigma)=1-(1+\beta x)^{-\sigma}$

$h(x ; \beta, \sigma)=\beta \sigma(1+\beta x)^{-(\sigma+1)}$

for $x \geq 0, \beta, \sigma>0$ where $\beta$ is the scale parameter and $\sigma$ is the shape parameter.

For some decades now, researchers have resorted to developing families of distributions and these families of distributions are proposed mainly to develop compound distributions. These compound probability distributions are expected to be more flexible and also fit data better than existing ones. Some of these generated families of distributions are: Topp Leone exponentiated-G by Ibrahim et al., (2020a), Topp Leone Kumaraswamy-G by Ibrahim et al., (2020b), The Kumaraswamy-G by (cordeiro and decastro, 2011), Topp Leone-G by Al-Shomrani et al., (2016), Odd Lindley-G family by GomesSilva et al., (2017), Gompertz-G family by Alizadeh et al., (2017), Odd Frechet G family by Haq and Elgarhy (2018), Power Lindley G family by Amal et al., (2017), Power Lindley-G Family of distributions by Hassan and Nassr (2019), Modi family of continuous probability distributions by Modi et al., (2020), etc. In this context, we proposed a generalization of the Lomax distribution based on Ibrahim et al., (2020b) which stems from the following general construction: if $H$ denotes the baseline cumulative function of a random variable, then a generalized class of distributions can be defined by $F(x ; \alpha, \lambda, \theta, \xi)=\left\{1-\left[1-H(x ; \xi)^{\alpha}\right]^{2 \lambda}\right\}^{\theta}$

The pdf corresponding to (3) is

$f(x ; \alpha, \lambda, \theta, \xi)=2 \alpha \lambda \theta h(x ; \xi) H(x ; \xi)^{\alpha-1}\left[1-H(x ; \xi)^{\alpha}\right]^{2 \lambda-1}\left\{1-\left[1-H(x ; \xi)^{\alpha}\right]^{2 \lambda}\right\}^{\theta-1}$

where $H(x ; \xi)$ is the cdf of the baseline distribution with parameter vector $\xi$.

For $x \geq 0, \alpha, \lambda, \theta, \xi \geq 0$, where equations (3) and (4) are the cdf and pdf of the TLK-G family of distributions. This paper proposes a new distribution that generalizes the Lomax distribution using the family of distribution proposed by Ibrahim et al., (2020). This is to improve the flexibility of the Lomax distribution to fit a variety of data including unimodal and bimodal shapes from different disciplines. The rest of the paper is outlined as follows. In section 2, we define the Topp Leone Kumaraswamy Lomax distribution, obtain some of its mathematical properties and distribution of order statistic. Estimation of the parameters using the method of Maximum likelihood estimation is performed in section 3. In section 4, real-life application of the distribution to data sets is provided. Section 5 presents 
concluding remarks. Acknowledgement is presented in section 6. Competing interest is presented in section 7 and finally, references are presented in section 8 .

\section{The Topp Leone Kumaraswamy Lomax (TLKLx) distribution}

In this section, we derive a new continuous distribution called TLKLx distribution that generalize and extend the Lomax distribution. The cdf of the new distribution is obtained by inserting (1) into (3) to get

$$
\begin{aligned}
& F(x ; \alpha, \theta, \beta, \lambda, \sigma)=\left\{1-\left[1-\left(1-(1+\beta x)^{-\sigma}\right)^{\alpha}\right]^{2 \lambda}\right\}^{\theta} \\
& f(x ; \alpha, \theta, \beta, \lambda, \sigma)=\frac{2 \alpha \theta \lambda \sigma \beta}{(1+\beta x)^{(\sigma+1)}}\left[1-(1+\beta x)^{-\sigma}\right]^{\alpha-1}\left[1-\left(1-(1+\beta x)^{-\sigma}\right)^{\alpha}\right]^{2 \lambda-1}\left\{1-\left[1-\left(1-(1+\beta x)^{-\sigma}\right)^{\alpha}\right]^{2 \lambda}\right\}^{\theta-1}
\end{aligned}
$$

for $x \geq 0, \alpha, \beta, \theta, \sigma, \lambda>0$, where $\alpha, \theta, \lambda$ are the shape parameters and $\beta$ is the scale parameter. The plots of pdf of the TLKLx distribution showing different shapes with different parameter values are presented in figure 1.
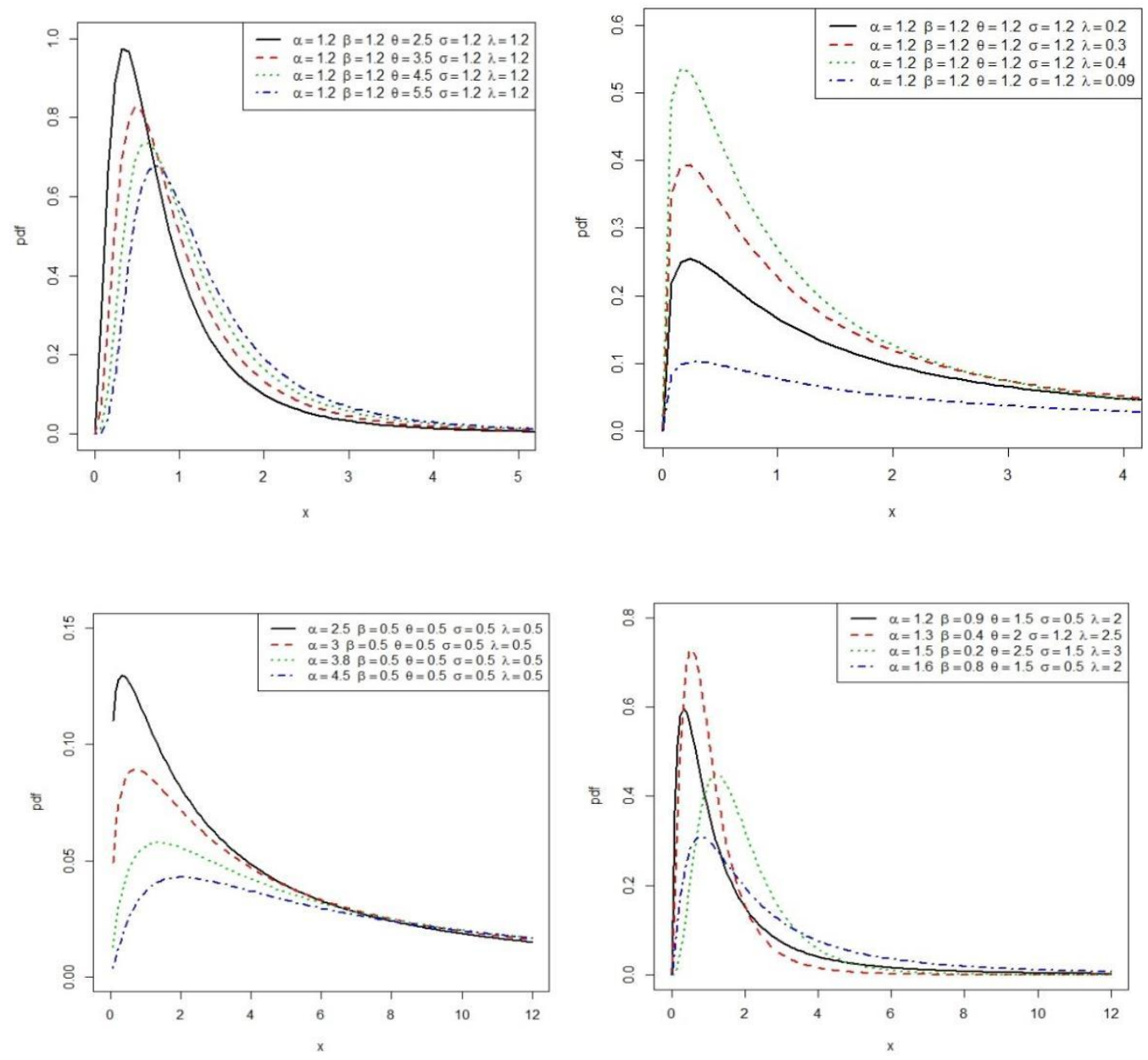

Figure 1: Plots of pdf of the TLKLx distribution for different parameter values.

\subsection{Properties of the TLKLx distribution}

This sub-section discusses the properties of the new models. 


\subsubsection{Moments}

It is always pertinent to keep in mind the importance of moments in any statistical analysis particularly in applied fields. For example, through moments the important characteristics such as tendency, dispersion, skewness, and kurtosis of a distribution can be studied.

Assume $Y$ is a Lomax distributed random variable with parameters $\beta$ and $\sigma$, then the $r^{\text {th }}$ moment of $Y$ is given as

$$
E\left(Y^{r}\right)=\left(\frac{\sigma}{\beta^{r}}\right) B(r+1, \sigma-r)
$$

Let $X$ be a random variable having the TLKLx distribution. Using the expansion in (7), it is easy to obtain the $r^{\text {th }}$ moment of $X$ as

$$
E\left(X^{r}\right)=2 \alpha \theta \lambda \sigma \beta Q\left(\frac{\sigma(i+1)}{\beta^{r}}\right) B(r+1, \sigma(i+1)-r)
$$

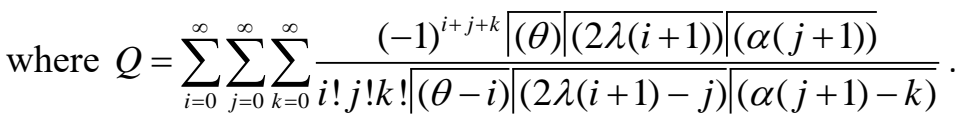

\subsubsection{Moment generating function}

The moment generating function (mgf) is another most prominent approach to study the behavior of probability distributions. A general expression for the MGF is given as

$M_{x}(t)=\int_{0}^{\infty} e^{t x} f(x) d x$

Since the series expansion for $e^{t x}$ is given as

$$
\begin{aligned}
& e^{t x}=\sum_{m=0}^{\infty} \frac{(t x)^{m}}{m !} \\
& M_{x}(t)=2 \alpha \theta \lambda \sigma \beta Z\left(\frac{\sigma(i+1)}{\beta^{r}}\right) B(m+1, \sigma(i+1)-m)
\end{aligned}
$$

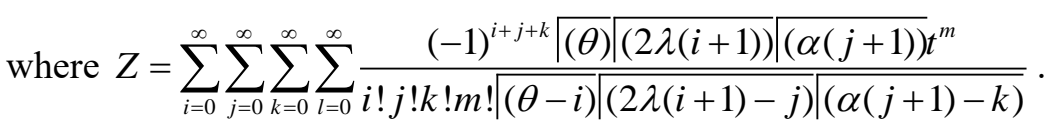

Substituting $r=1,2,3,4$ in (8), we obtain the mean $=\mu_{1}^{\prime}$, variance $=\mu_{2}^{\prime}-\mu_{1}^{\prime 2}$, skewness $=\frac{\mu_{3}^{\prime}}{\mu_{1}^{\frac{3}{2}}}$ and kurtosis $=\frac{\mu_{4}^{\prime}}{\mu_{1}^{\prime 2}}$.

\subsubsection{Reliability}

The reliability function is also known as survival function, which is the probability of an item not failing prior to some time. It can be defined as 


$$
R(x ; \alpha, \theta, \beta, \lambda, \sigma)=1-F(x ; \alpha, \theta, \beta, \lambda, \sigma)
$$

The survival function $R(x)$ of TLKLx distribution is given as

$$
R(x ; \alpha, \theta, \beta, \lambda, \sigma)=1-\left\{1-\left[1-\left(1-(1+\beta x)^{-\sigma}\right)^{\alpha}\right]^{2 \lambda}\right\}^{\theta}
$$

\subsubsection{Hazard rate function}

The hazard rate function (hrf) is defined as

$$
\tau(x ; \alpha, \theta, \beta, \lambda, \sigma)=\frac{f(x ; \alpha, \theta, \beta, \lambda, \sigma)}{R(x ; \alpha, \theta, \beta, \lambda, \sigma)}
$$

Then, the hrf $\tau(x)$ of the TLKLx distribution is obtained as

$$
\tau(x ; \alpha, \theta, \beta, \lambda, \sigma)=\frac{2 \alpha \theta \lambda \sigma \beta(1+\beta x)^{-(\sigma+1)}\left[1-(1+\beta x)^{-\sigma}\right]^{\alpha-1}\left[1-\left(1-(1+\beta x)^{-\sigma}\right)^{\alpha}\right]^{2 \lambda-1}\left\{1-\left[1-\left(1-(1+\beta x)^{-\sigma}\right)^{\alpha}\right]^{2 \lambda}\right\}^{\theta-1}}{1-\left\{1-\left[1-\left(1-(1+\beta x)^{-\sigma}\right)^{\alpha}\right]^{2 \lambda}\right\}^{\theta}}
$$
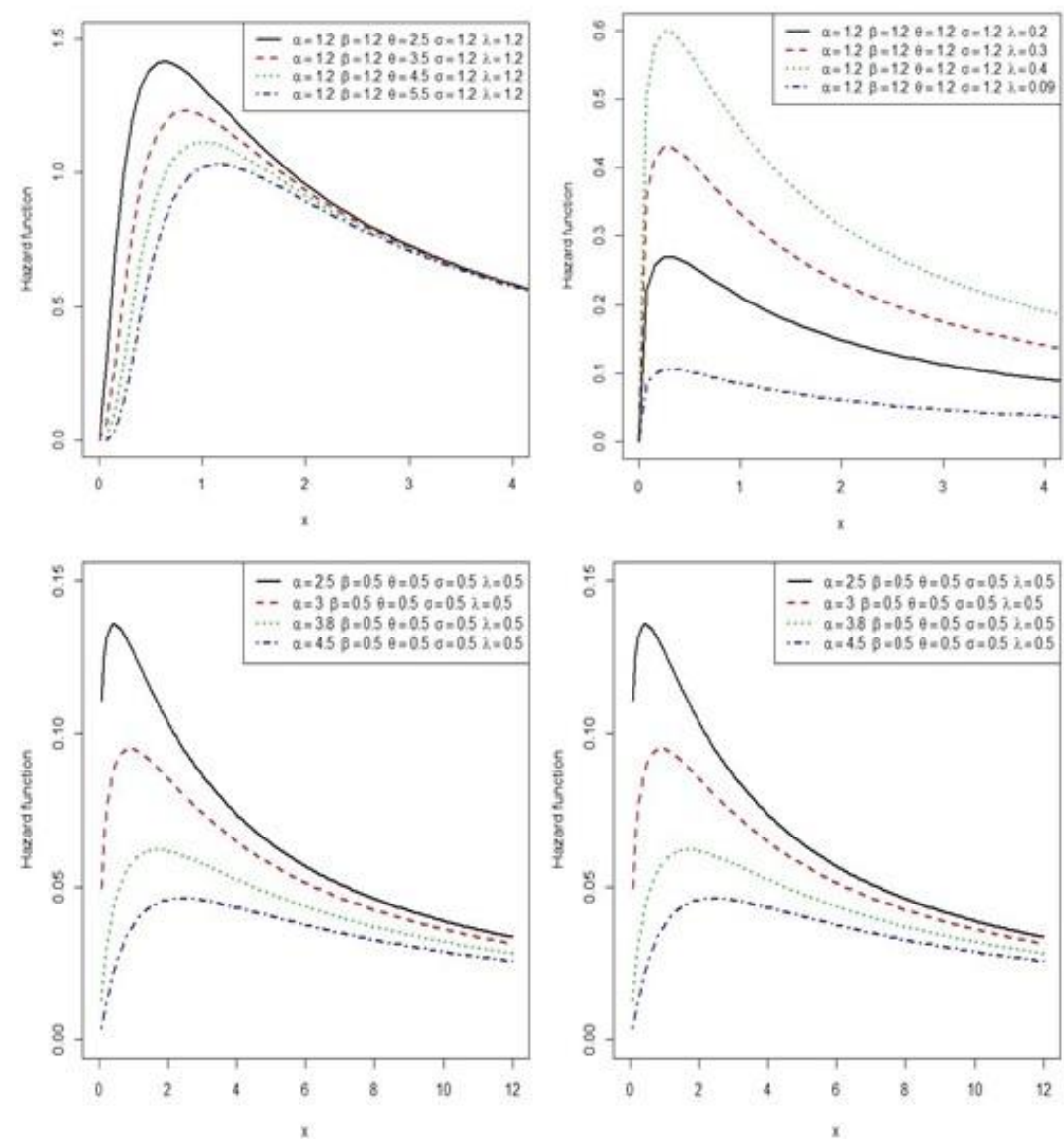

Figure 2. Plots of the hazard function of the TLKLx distribution for different parameter values.

Figure 1 and 2 show the shapes of the new model. It can be seen from the plots that the new model has different shapes like increasing, decreasing, skewed, J-shape, reversed J-shape and bathtub which makes it more flexible in fitting different kinds of real life data. 


\subsubsection{Reversed hazard rate function}

The reversed hazard rate function for the TLKLx distribution is obtained by using the relation

$$
\begin{aligned}
& r(x ; \alpha, \theta, \beta, \lambda, \sigma)=\frac{f(x ; \alpha, \theta, \beta, \lambda, \sigma)}{F(x ; \alpha, \theta, \beta, \lambda, \sigma)} \\
& r(x ; \alpha, \theta, \beta, \lambda, \sigma)=\frac{2 \alpha \theta \lambda \sigma \beta(1+\beta x)^{-(\sigma+1)}\left[1-(1+\beta x)^{-\sigma}\right]^{\alpha-1}\left[1-\left(1-(1+\beta x)^{-\sigma}\right)^{\alpha}\right]^{2 \lambda-1}\left\{1-\left[1-\left(1-(1+\beta x)^{-\sigma}\right)^{\alpha}\right]^{2 \lambda}\right\}^{\theta-1}}{\left\{1-\left[1-\left(1-(1+\beta x)^{-\sigma}\right)^{\alpha}\right]^{2 \lambda}\right\}^{\theta}}
\end{aligned}
$$

\subsubsection{Odds function}

The odds function for the TLExLx distribution is obtained as

$$
\begin{aligned}
& Q(x ; \alpha, \theta, \beta, \lambda, \sigma)=\frac{F(x ; \alpha, \theta, \beta, \lambda, \sigma)}{R(x ; \alpha, \theta, \beta, \lambda, \sigma)} \\
& Q(x ; \alpha, \theta, \beta, \lambda, \sigma)=\frac{\left\{1-\left[1-\left(1-(1+\beta x)^{-\sigma}\right)^{\alpha}\right]^{2 \lambda}\right\}^{\theta}}{1-\left\{1-\left[1-\left(1-(1+\beta x)^{-\sigma}\right)^{\alpha}\right]^{2 \lambda}\right\}^{\theta}}
\end{aligned}
$$

\subsubsection{Quantile function}

The quantile function is defined as the inverse of the cdf and it is given as $Q(u)=F^{-1}(u)$. Using the cdf of TLKLx distribution in (5), we have

$$
\begin{aligned}
& F(x ; \alpha, \theta, \beta, \lambda, \sigma)=\left\{1-\left[1-\left(1-(1+\beta x)^{-\sigma}\right)^{\alpha}\right]^{2 \lambda}\right\}^{\theta}=u \\
& u^{\frac{1}{\theta}}=1-\left[1-\left(1-(1+\beta x)^{-\sigma}\right)^{\alpha}\right]^{2 \lambda} \\
& 1-u^{\frac{1}{\theta}}=\left[1-\left(1-(1+\beta x)^{-\sigma}\right)^{\alpha}\right]^{2 \lambda} \\
& {\left[1-u^{\frac{1}{\theta}}\right]^{\frac{1}{2 \lambda}}=1-\left(1-(1+\beta x)^{-\sigma}\right)^{\alpha}} \\
& 1-\left[1-u^{\frac{1}{\theta}}\right]^{\frac{1}{2 \lambda}}=\left(1-(1+\beta x)^{-\sigma}\right)^{\alpha} \\
& {\left[1-\left[1-u^{\frac{1}{\theta}}\right]^{\frac{1}{2 \lambda}}\right]^{\frac{1}{\alpha}}=1-(1+\beta x)^{-\sigma}}
\end{aligned}
$$




$$
\begin{aligned}
& 1-\left[1-\left[1-u^{\frac{1}{\theta}}\right]^{\frac{1}{2 \lambda}}\right]^{\frac{1}{\alpha}}=(1+\beta x)^{-\sigma} \\
& {\left[1-\left[1-\left[1-u^{\frac{1}{\theta}}\right]^{\frac{1}{2 \lambda}}\right]^{\frac{1}{\alpha}}\right]^{\frac{1}{-\sigma}}=1+\beta x} \\
& {\left[1-\left[1-\left[1-u^{\frac{1}{\theta}}\right]^{\frac{1}{2 \lambda}}\right]^{\frac{1}{\alpha}}\right]^{\frac{1}{-\sigma}}-1=\beta x} \\
& x=\frac{1}{\beta}\left\{\left[1-\left[1-\left[1-u^{\frac{1}{\theta}}\right]^{\frac{1}{2 \lambda}}\right]^{\frac{1}{\alpha}}-1\right\}\right.
\end{aligned}
$$

Using the equation (20), we can generate random sample from the TLKLx distribution by using $U$ as uniform random number. The median of the TLKLx distribution can be derived by substituting $u=0.5$ in (20) as follows:

$$
\text { median }=\frac{1}{\beta}\left\{\left[1-\left[1-\left[1-0.5^{\frac{1}{\theta}}\right]^{\frac{1}{2 \lambda}}\right]^{\frac{1}{\alpha}}\right]^{\frac{1}{-\sigma}}-1\right\}
$$

\section{$2.2 \quad$ Order statistic}

Let $X_{1}, X_{2}, \ldots, X_{n}$ be $n$ independent random variable from the TLKLx distributions and let $X_{(1)} \leq X_{(2)} \leq \ldots \leq X_{(n)}$ be their corresponding order statistic. Let $F_{r: n}(x)$ and $f_{r: n}(x), r=1,2,3, \ldots n$ denote the cdf and pdf of the $r^{\text {th }}$ order statistics $X_{r: n}$ respectively. The pdf of the $r^{\text {th }}$ order statistics of $X_{r: n}$ is given as

$$
f_{r: n}(x)=\frac{1}{B(r, n-r+1)} \sum_{i=0}^{\infty} \frac{(-1)^{i} \sqrt{(n-r+1)}}{i !(n-r+1-i)}[F(x)]^{r+i-1} f(x)
$$

Using the cdf and pdf of TLKLx distribution in (5) and (6), we have

$$
f_{r n}(x)=\frac{2 \alpha \beta \theta \lambda \sigma}{B(r, n-r+1)} \sum_{i=0}^{\infty} \frac{(-1)^{i}[(n-r+1)}{i !(n-r+1-i)}\left\{1-\left[1-\left(1-(1+\beta x)^{-\sigma}\right)^{\alpha}\right]^{2 \lambda}\right\}^{\theta(r+i)-1}(1+\beta x)^{-(\sigma+1)}\left[1-(1+\beta x)^{-\sigma}\right]^{\alpha-1}\left[1-\left(1-(1+\beta x)^{-\sigma}\right)^{\alpha}\right]^{2 \lambda-1}
$$

Equation (23) is the $r^{\text {th }}$ order statistic of TLKLx distribution. To obtain the minimum order statistic, we 
set $r=1$ in (23) to get

$$
f_{r n}(x)=\frac{2 \alpha \beta \theta \lambda \sigma \sigma}{B(1, n)} \sum_{i=0}^{\infty} \frac{(-1)^{i}(\overline{l n})}{i !(n-i)}\left\{1-\left[1-\left(1-(1+\beta x)^{-\sigma}\right)^{\alpha}\right]^{2 \lambda}\right\}^{\theta(r+i)-1}(1+\beta x)^{-(\sigma+1)}\left[1-(1+\beta x)^{-\sigma}\right]^{\alpha-1}\left[1-\left(1-(1+\beta x)^{-\sigma}\right)^{\alpha}\right]^{2 \lambda-1}
$$

Using binomial expansion on

$$
\begin{aligned}
& \left\{1-\left[1-\left(1-(1+\beta x)^{-\sigma}\right)^{\alpha}\right]^{2 \lambda}\right\}^{\theta(r+i)-1}=\sum_{j=0}^{\infty} \frac{(-1)^{j} \sqrt{(\theta(1+i))}}{j !(\theta(1+i)-j)}\left[1-\left(1-(1+\beta x)^{-\sigma}\right)^{\alpha}\right]^{2 \lambda j} \\
& {\left[1-\left(1-(1+\beta x)^{-\sigma}\right)^{\alpha}\right]^{2 \lambda(j+1)-1}=\sum_{k=0}^{\infty} \frac{(-1)^{k} \sqrt{k !(2 \lambda(j+1))}}{k !(2 \lambda(j+1)-k)}\left(1-(1+\beta x)^{-\sigma}\right)^{\alpha k}} \\
& \left(1-(1+\beta x)^{-\sigma}\right)^{\alpha(k+1)-1}=\sum_{l=0}^{\infty} \frac{(-1)^{l} \sqrt{(\alpha(k+1))}}{l !(\alpha(k+1)-l)}(1+\beta x)^{-\sigma l}
\end{aligned}
$$

Therefore, the minimum order statistics of TLKLx distribution is given as

$$
f_{1: n}(x)=2 n \alpha \beta \theta \lambda \sigma \sum_{i=0}^{\infty} \sum_{j=0}^{\infty} \sum_{k=0}^{\infty} \sum_{l=0}^{\infty} \frac{(-1)^{i+j+k+l} \sqrt{n} \sqrt{(\theta(i+1))} \sqrt{(2 \lambda(j+1))} \sqrt{(\alpha(k+1))}}{(1, k ! l !|n-i|[(\theta(i+1)-j)}(1+\beta x)^{-\sigma(l+1)-1}
$$

Also, the maximum order statistics of TLKLx distribution is given as

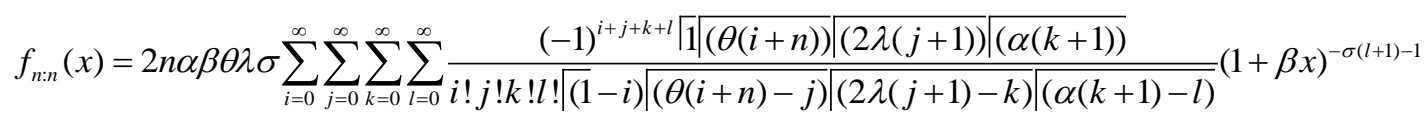

\section{Estimation}

In this section, we estimate the parameters of the TLKLx distribution using maximum likelihood estimation (MLE). For a random sample, $X_{1}, X_{2}, \ldots, X_{n}$ of size $n$ from the TLKLx $(\alpha, \beta, \theta, \lambda, \sigma)$, the $\log$-likelihood function $L(\alpha, \beta, \theta, \lambda, \sigma)$ of (6) is given as

$$
\begin{aligned}
\log L= & n \log 2+n \log \alpha+n \log \sigma+n \log \lambda+n \log \theta-(\sigma+1) \sum_{i=1}^{n} \log \left[1+\beta x_{i}\right]+(\alpha-1) \sum_{i=1}^{n}\left[1-\left(1+\beta x_{i}\right)^{-\sigma}\right] \\
& +(2 \lambda-1) \sum_{i=1}^{n} \log \left[1-\left(1-\left(1+\beta x_{i}\right)^{-\sigma}\right)^{\alpha}\right]+(\theta-1) \sum_{i=1}^{n} \log \left[1-\left(1-\left(1-\left(1+\beta x_{i}\right)^{-\sigma}\right)^{\alpha}\right)^{2 \lambda}\right]
\end{aligned}
$$

Differentiating the log-likelihood with respect to $\alpha, \beta, \theta, \lambda, \sigma$ and setting the result equals to zero, we have

$$
\begin{aligned}
\frac{\partial \log L}{\partial \alpha} & =\frac{n}{\alpha}+\sum_{i=1}^{n}\left[1-\left(1+\beta x_{i}\right)^{-\sigma}\right]+(2 \lambda-1) \sum_{i=1}^{n} \frac{\left[1-\left(1+\beta x_{i}\right)^{-\sigma}\right]^{\alpha} \log \left[1-\left(1+\beta x_{i}\right)^{-\sigma}\right]}{1-\left[1-\left(1+\beta x_{i}\right)^{-\sigma}\right]^{\alpha}} \\
& +(\theta-1) \sum_{i=1}^{n} \frac{2 \lambda \alpha\left[1-\left(1-\left(1+\beta x_{i}\right)^{-\sigma}\right)^{\alpha}\right]^{2 \lambda-1}\left[1-\left(1+\beta x_{i}\right)^{-\sigma}\right]^{\alpha} \log \left[1-\left(1+\beta x_{i}\right)^{-\sigma}\right]^{\alpha}}{1-\left[1-\left(1-\left(1+\beta x_{i}\right)^{-\sigma}\right)^{\alpha}\right]^{2 \lambda}}=0
\end{aligned}
$$




$$
\begin{aligned}
\frac{\partial \log L}{\partial \beta} & =\frac{n}{\beta}-(\sigma+1) \sum_{i=1}^{n} \frac{x_{i}}{\left(1+\beta x_{i}\right)}+(\alpha-1) \sum_{i=1}^{n} \frac{\sigma x_{i}\left(1+\beta x_{i}\right)^{-\sigma-1}}{\left[1-\left(1+\beta x_{i}\right)^{-\sigma}\right]}+(2 \lambda-1) \sum_{i=1}^{n} \frac{\alpha\left[1-\left(1+\beta x_{i}\right)^{-\sigma}\right]^{\alpha-1} \sigma x_{i}\left(1+\beta x_{i}\right)^{-\sigma-1}}{\left[1-\left(1-\left(1+\beta x_{i}\right)^{-\sigma}\right)^{\alpha}\right]} \\
& +(\theta-1) \sum_{i=1}^{n} \frac{2 \lambda\left[1-\left(1-\left(1+\beta x_{i}\right)^{-\sigma}\right)^{\alpha}\right]^{2 \lambda-1} \alpha\left[1-\left(1+\beta x_{i}\right)^{-\sigma}\right]^{\alpha-1} \sigma x_{i}\left(1+\beta x_{i}\right)^{-\sigma-1}}{\left[1-\left[1-\left(1-\left(1+\beta x_{i}\right)^{-\sigma}\right)^{\alpha}\right]^{2 \lambda}\right]}=0
\end{aligned}
$$

$$
\begin{aligned}
\frac{\partial \log L}{\partial \sigma} & =\frac{n}{\sigma}-\sum_{i=1}^{n} \log \left[1+\beta x_{i}\right]+(\alpha-1) \sum_{i=1}^{n} \frac{\left(1+\beta x_{i}\right)^{-\sigma} \log \left(1+\beta x_{i}\right)^{-\sigma}}{\left[1-\left(1+\beta x_{i}\right)^{-\sigma}\right]} \\
& +(\theta-1) \sum_{i=1}^{n} \frac{2 \lambda \alpha\left[1-\left(1-\left(1+\beta x_{i}\right)^{-\sigma}\right)^{\alpha}\right]^{2 \lambda-1}\left[1-\left(1+\beta x_{i}\right)^{-\sigma}\right]^{\alpha-1}\left(1+\beta x_{i}\right)^{-\sigma} \log \left(1+\beta x_{i}\right)^{-\sigma}}{\left[1-\left[1-\left(1-\left(1+\beta x_{i}\right)^{-\sigma}\right)^{\alpha}\right]^{2 \lambda}\right]} \\
& +(2 \lambda-1) \sum_{i=1}^{n} \frac{\alpha\left[1-\left(1+\beta x_{i}\right)^{-\sigma}\right]^{\alpha-1}\left(1+\beta x_{i}\right)^{-\sigma} \log \left(1+\beta x_{i}\right)^{-\sigma}}{\left[1-\left(1-\left(1+\beta x_{i}\right)^{-\sigma}\right)^{\alpha}\right]}=0
\end{aligned}
$$

$\frac{\partial \log L}{\partial \lambda}=\frac{n}{\lambda}+2 \sum_{i=1}^{n} \log \left[1-\left(1-\left(1+\beta x_{i}\right)^{-\sigma}\right)^{\alpha}\right]+(\theta-1) \sum_{i=1}^{n}\left[\frac{\left[1-\left(1-\left(1+\beta x_{i}\right)^{-\sigma}\right)^{\alpha}\right]^{2 \lambda} \log \left[1-\left(1-\left(1+\beta x_{i}\right)^{-\sigma}\right)^{\alpha}\right]}{1-\left[1-\left(1-\left(1+\beta x_{i}\right)^{-\sigma}\right)^{\alpha}\right]^{2 \lambda}}\right]=0$

$$
\frac{\partial \log L}{\partial \theta}=\frac{n}{\theta}+\sum_{i=1}^{n} \log \left[1-\left[1-\left(1-\left(1+\beta x_{i}\right)^{-\sigma}\right)^{\alpha}\right]^{2 \lambda}\right]=0
$$

Now, equations (31), (32), (33), (34) and (35) do not have a simple form and are therefore intractable. As a result, we have to resort to non-linear estimation of the parameters using iterative procedures.

\section{Application to real-life data}

The first data set represents the failure times of the air conditioning system of an airplane. The data set was given by Linhart and Zucchini (1986) and it has also been used by Shanker et al., (2015). It has thirty (30) observations as follows: $23,261,87,7,120,14,62,47,225,71,246,21,42,20,5,12,120$, $11,3,14,71,11,14,11,16,90,1,16,52,95$.

The second data set was given by Lee and Wang (2003) and it represents the remission times (in months) of a random sample of one hundred and twenty-eight (128) bladder cancer patients. The data set is given as:

$0.08,2.09,3.48,4.87,6.94,8.66,13.11,23.63,0.20,2.23,3.52,4.98,6.97,9.02,13.29,0.40,2.26$, $3.57,5.06,7.09,9.22,13.80,25.74,0.50,2.46,3.64,5.09,7.26,9.47,14.24,25.82,0.51,2.54,3.70$, $5.17,7.28,9.74,14.76,26.31,0.81,2.62,3.82,5.32,7.32,10.06,14.77,32.15,2.64,3.88,5.32,7.39$, $10.34,14.83,34.26,0.90,2.69,4.18,5.34,7.59,10.66,15.96,36.66,1.05,2.69,4.23,5.41,7.62,10.75$, $16.62,43.01,1.19,2.75,4.26,5.41,7.63,17.12,46.12,1.26,2.83,4.33,5.49,7.66,11.25,17.14,79.05$, $1.35,2.87,5.62,7.87,11.64,17.36,1.40,3.02,4.34,5.71,7.93,11.79,18.10,1.46,4.40,5.85,8.26$, $11.98,19.13,1.76,3.25,4.50,6.25,8.37,12.02,2.02,3.31,4.51,6.54,8.53,12.03,20.28,2.02,3.36$, $6.76,12.07,21.73,2.07,3.36,6.93,8.65,12.63,22.69$.

The third data is Alumina (A12O3) taken from the website: http://www.ceramics.nist.gov/srd/summary/ftmain.htm. This data set can also be found in Nadarajah 
and Kotz (2008) and the data set is given as:

$5.5,5,4.9,6.4,5.1,5.2,5.2,5,4.7,4,4.5,4.2,4.1,4.56,5.01,4.7,3.13,3.12,2.68,2.77,2.7,2.36,4.38$, $5.73,4.35,6.81,1.91,2.66,2.61,1.68,2.04,2.08,2.13,3.8,3.73,3.71,3.28,3.9,4,3.8,4.1,3.9,4.05$, $4,3.95,4,4.5,4.5,4.2,4.55,4.65,4.1,4.25,4.3,4.5,4.7,5.15,4.3,4.5,4.9,5,5.35,5.15,5.25,5.8$, $5.85,5.9,5.75,6.25,6.05,5.9,3.6,4.1,4.5,5.3,4.85,5.3,5.45,5.1,5.3,5.2,5.3,5.25,4.75,4.5,4.2$, $4,4.15,4.25,4.3,3.75,3.95,3.51,4.13,5.4,5,2.1,4.6,3.2,2.5,4.1,3.5,3.2,3.3,4.6,4.3,4.3,4.5,5.5$, $4.6,4.9,4.3,3,3.4,3.7,4.4,4.9,4.9,5$

The competing models used in this analysis are Kumaraswamy Exponentiated Lomax (KExLx) distribution with five parameters, Exponentiated Lomax (ExLx) distribution with four parameters and Lomax (Lx) distribution with two parameters.

- Kumaraswamy Exponentiated Lomax (KExLx)distribution (El-Batal and Kareem 2014)

$$
f(x)=\alpha \theta \beta \lambda(1+\beta x)^{-(\sigma+1)}\left[1-(1+\beta x)^{-\sigma}\right]^{\alpha \lambda-1}\left[1-\left[1-(1+\beta x)^{-\sigma}\right]^{\alpha \lambda}\right]^{\theta-1}
$$

- ExLx distribution (Salem, 2014)

$$
f(x)=\theta \sigma \beta(1+\beta x)^{-(\sigma+1)}\left[1-(1+\beta x)^{-\sigma}\right]^{\theta-1}
$$

- Lx distribution

$$
\sigma \beta(1+\beta x)^{-(\sigma+1)}
$$

Table 1. The MLEs and Information Criteria of the models based on data set 1 .

\begin{tabular}{|c|c|c|c|c|c|c|c|c|c|}
\hline Models & $\hat{\alpha}$ & $\hat{\beta}$ & $\hat{\theta}$ & $\hat{\sigma}$ & $\hat{\lambda}$ & $-l$ & AIC & BIC & CAIC \\
\hline TLKLx & 0.2743 & 0.0004 & 7.3247 & 2.6739 & 2.3638 & 151.3074 & 312.6148 & 319.6208 & 315.1148 \\
\hline KExLx & 1.4565 & 0.0126 & 0.4519 & 5.1476 & 0.8470 & 151.7755 & 313.5511 & 320.5571 & 316.0511 \\
\hline ExLx & - & 0.5889 & 5.5254 & 0.7859 & - & 154.2919 & 314.5837 & 318.7873 & 315.5068 \\
\hline Lx & - & 0.0587 & - & 0.7014 & - & 155.3782 & 314.7563 & 317.5587 & 315.2008 \\
\hline
\end{tabular}

Table 2. The MLEs and Information Criteria of the models based on data set 2 .

\begin{tabular}{|c|c|c|c|c|c|c|c|c|c|}
\hline Models & $\hat{\alpha}$ & $\hat{\beta}$ & $\hat{\theta}$ & $\hat{\sigma}$ & $\hat{\lambda}$ & $-l$ & AIC & BIC & CAIC \\
\hline TLKLx & 3.431 & 0.131 & 0.463 & 0.899 & 2.463 & 410.065 & 830.129 & 844.389 & 830.622 \\
\hline KExLx & 0.491 & 0.107 & 0.3482 & 5.244 & 3.430 & 415.842 & 841.685 & 855.945 & 842.176 \\
\hline ExLx & - & 6.823 & 15.133 & 0.871 & - & 439.873 & 885.746 & 894.302 & 885.939 \\
\hline Lx & - & 0.009 & - & 13.439 & - & 413.834 & 831.667 & 837.371 & 831.763 \\
\hline
\end{tabular}


Table 3. The MLEs and Information Criteria of the models based on data set 3 .

\begin{tabular}{|c|c|c|c|c|c|c|c|c|c|}
\hline Models & $\hat{\alpha}$ & $\hat{\beta}$ & $\hat{\theta}$ & $\hat{\sigma}$ & $\hat{\lambda}$ & $-l$ & AIC & BIC & CAIC \\
\hline TLKLx & 20.754 & 0.024 & 0.399 & 13.832 & 38.567 & 169.016 & 348.032 & 361.927 & 348.563 \\
\hline KExLx & 36.909 & 0.594 & 40.656 & 1.454 & 0.6312 & 175.241 & 360.482 & 374.378 & 361.013 \\
\hline ExLx & - & 21.767 & 14.765 & 0.062 & - & 194.824 & 395.649 & 403.986 & 395.857 \\
\hline Lx & - & 0.0585 & - & 4.099 & - & 306.111 & 616.222 & 621.780 & 616.326 \\
\hline
\end{tabular}

The tables 1-3 present the estimate of each parameter and goodness of fit for the models considered. The goodness of fits considered are the Akaike Information Criteria (AIC), Bayessian Information Criteria (BIC) and Corrected Akaike Information Criteria (CAIC). The smaller the AIC, BIC and CAIC values the better the model.

The figures 3-5 present the shapes, fit and flexibility of the new model in relation to the data sets considered. The black line represents the new model, the red line represents the KExLx, the green line represents the ExLx while the blue line represents the Lx distributions. It can be seen from the plots the the black line which represents the TLKLx distribution fits better in the three data sets considered.

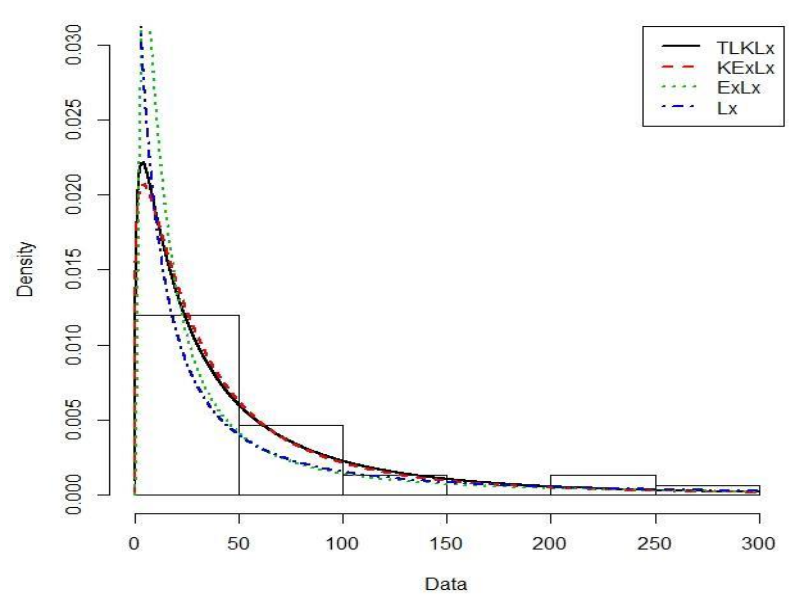

Figure 3: Fitted pdfs of the TLKLx, KExLx, ExLx and Lx models for data set 1. 


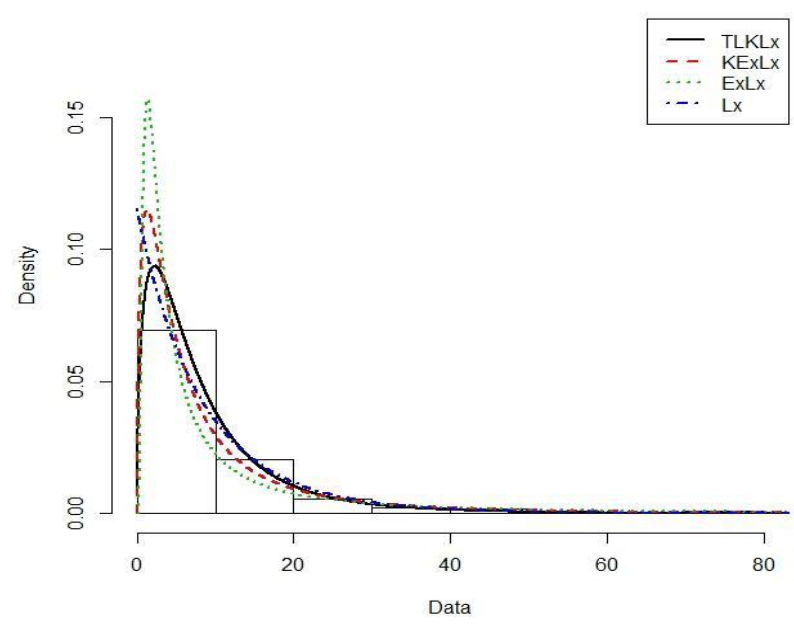

Figure 4: Fitted pdfs of the TLKLx, KExLx, ExLx and Lx models for data set 2.

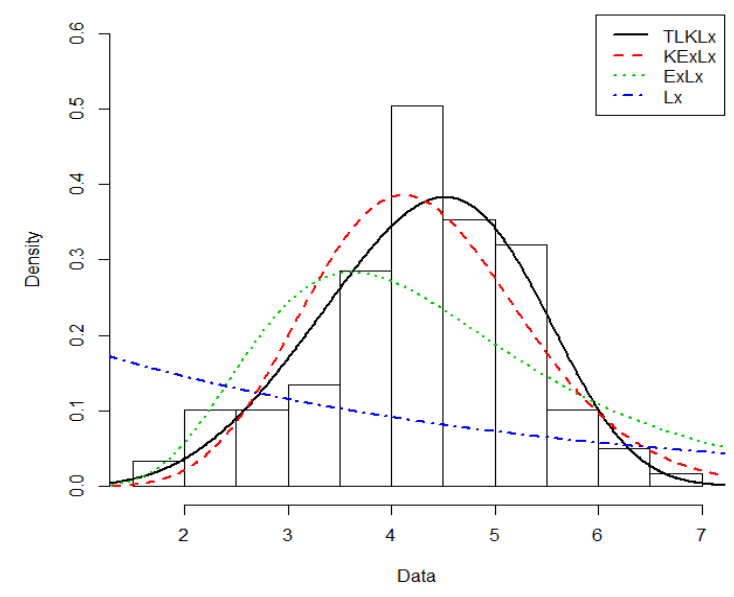

Figure 5: Fitted pdfs of the TLKLx, KExLx, ExLx and Lx models for data set 3.

\section{Conclusion}

This paper has developed a new distribution called the Topp Leone Kumaraswamy Lomax distribution that generalizes the Lomax distribution. Some properties of the new distribution were derived such as the survival function, hazard rate function, quantile function, the median and order statistics. The shapes of the proposed distribution were shown by plotting the graphs of the pdf and hazard function. The estimation of the model parameters by the method of the maximum likelihood was carried out using a package in $R$ known as AdequacyModel. Application of the Topp Leone Kumaraswamy Lomax distribution to three real datasets shown from Table 1, Table 2 and Table 3 that the Topp Leone Kumaraswamy Lomax distribution is quite effective and superior in fitting the three data sets considered. Also, from the fitted pdf plots for the three data sets, it can be deduced that the new models fit the three data sets better than the competing models considered. 


\section{Acknowledgements}

The authors are grateful to the Editor-in-Chief, the Associate Editor and anonymous reviewers for their constructive comments and suggestions which led to remarkable improvement of the paper.

\section{Competing interests}

The authors declare that they have no competing interests.

\section{References}

Abdul-Moniem, I. B. and Abdel-Hameed, H. F. (2012). On exponentiated Lomax distribution, International Journal of Mathematical Archive, 3: 2144-2150.

Alizadeh, M., Cordeiro, G. M, Pinho, L. G. B. and Ghosh I. (2017). The Gompertz-G family of distributions. Journal of Statistical Theory and Practice, 11(1):179-207.

Al-Shomrani, A., Arif, O., Shawky, A., Hanif, S. and Shahbaz, M. Q. (2016). Topp-Leone family of distributions: Some properties and application. Pakistan Journal of Statistics and Operation Research, XII, 3: 443-451.

Brito, E., Cordeiro, G. M., Yousof, H. M., Alizadeh, M., and Silva, G. O. (2017). The Topp Leone odd log-logistic family of distributions. Journal of Statistical Computation and Simulation, 87(15): 3040-3058.

Cordeiro, G. M., and de Castro, M. (2011). A new family of generalized distributions, Journal of Statistical Computation and Simulation, 81(7): 883-898.

Cordeiro, G., Ortega E. and Popovic, B. (2013). The gamma Lomax distribution. Journal of Statistical Computation and Simulation; 85(2): 305-319

El-Bassiouny, A. H., Abdo, N. F. and Shahen, H. S. (2015). Exponential Lomax distribution. International Journal of Computer applications, 121(13): 24-29.

El-Batal, I. and Kareem, A. (2014). Statistical properties of kumaraswamy exponentiated Lomax distribution, Journal of Modern Mathematics and Statistics, 8(1): 1-7.

Gomes-Silva, F. S., Percontini, A., de Brito, E., Ramos, M. W., Venancio, R. and Cordeiro, G. M. (2017). The odd Lindley-G family of distributions. Austrian Journal of Statistics, 46(1): 65-87.

Haq, M. A. and Elgarhy, M. (2018). The odd Frechet-G family of probability distributions. Journal of Statistics Applications and Probability, 7(1): 189-203.

Hassan, A. S. and Nassr, S. G. (2019). Power Lindley-G family of distributions. Annals of Data Science, 6(2):189-210.

Ibrahim, S., Doguwa S. I., Audu, I. and Jibril, H. M. (2020a). On the Topp Leone exponentiated-G Family of Distributions: Properties and Applications. Asian Journal of Probability and Statistics, 7(1): $1-15$.

Ibrahim, S., Doguwa S. I., Audu, I. and Jibril, H. M. (2020b). The Topp Leone Kumaraswamy-G Family of Distributions with Applications to Cancer Disease Data, Journal of Biostatistics and 
Epidemiology, 6(1): 37-48.

Lee, E. T. and Wang, J. W. (2003). Statistical methods for survival data analysis (3rd Edition), John Wiley and Sons, New York, USA, 535 Pages, ISBN 0-471-36997-7.

Lemonte, A. and Cordeiro, G. (2013). An extended Lomax distribution. Statistics, 47: 800-816.

Linhart, H. and Zucchini, W. (1986). Model selection, John Wiley, New York, USA; [ISBN: 0-47183722-9]

Lomax, K. S. (1954). Business failures: Another example of the analysis of failure data, Journal of the American Statistical Association, 49: 847-852

Nadarajah, S. and Kotz, S. (2008). Strength modeling using Weibull distributions, J. Mech. Sci. Tech., 22(8): 1247-1254. 